\title{
Capital humano, teorías y métodos: importancia de la variable salud
}

\section{Human capital, theories and methods: importance of the health variable}

\author{
Dewin Iván Pérez-Fuentes* \\ Jorge Leonardo Castillo-Loaiza**
}

\begin{abstract}
This work presents a literature review on the concept of human capital, showing the different measurement methodologies; the relation that it maintains with labor market and health, and some facts stylized on this matter in Latin America and several regions of the world. The classic school provided the first expositions; later they were formalized by Schultz, and from then on several authors continued the legacy. Empirical analyses demonstrated that the level of human capital is correlated positively with the health variable, and exhibits big differences at regional level, which favor Western Europe and North America.
\end{abstract}

Keywords: human capital, labor market, health, mortality.

\section{Resumen}

Este trabajo presenta una revisión de literatura sobre el concepto de capital humano, se muestran las diferentes metodologías de medición, la relación que mantiene con el mercado laboral y la salud, y algunos hechos estilizados al respecto en América Latina y varias regiones del mundo. Los primeros planteamientos los proporcionó la escuela clásica, posteriormente fueron formalizados por Schultz, y en adelante varios autores continuaron el legado. Los análisis empíricos demostraron que el nivel de capital humano se correlaciona positivamente con la variable salud y exhibe grandes diferencias a nivel regional, las cuales favorecen a Europa occidental y América del Norte.

Palabras clave: capital humano, mercado laboral, salud, mortalidad.

\footnotetext{
* Programa de Economía de la Universidad de Cartagena, Colombia. Correo-e: dewinperez@ yahoo.es

** Departamento Administrativo de Ciencia Tecnología e Innovación de Colombia, Colombia. Correo-e: jleonardocasti@hotmail.com
} 


\section{Introducción}

A lo largo de la historia del pensamiento económico se ha olvidado casi completamente el estudio de una variable primordial en todas las economías: el capital humano. Hasta mediados del siglo XVIII, la influencia intelectual de los mercantilistas conllevaba a cuantificar la riqueza de una nación en función de una balanza comercial positiva. ${ }^{1}$ Más tarde, los fisiócratas se enfocaron en la agricultura como principal determinante de la riqueza de un país, basándose en la naturaleza como sustento básico y fundamental de la actividad económica (Domínguez, 2004). A estas teorías se agregaron las de la escuela clásica, las cuales le proporcionaron gran valor al sector industrial y a los mecanismos de fijación de precios en el mercado (Quezada, 2011).

El primer economista clásico que implementó el concepto capital bumano fue Adam Smith, quien en su obra pionera La riqueza de las naciones, propuso una similitud entre los hombres y las máquinas productoras. Más tarde, otros autores consideraron el concepto implementado por Smith, entre ellos Mill y Bentham, hasta la contribución de Marshall. La concepción del capital humano, a pesar de ser considerada por autores como Petty, Cantillon, Marshall, Fisher y Von Thuner, nunca fue desarrollada en el marco de una estructura teórica sólida, al menos hasta la mitad del siglo XX, gracias a las obras pioneras de Mincer (1958), Schultz (1960) y Becker (1964). Dichas obras aportaron una teoría económica sobre el capital humano que se basaba principalmente en los años de escolaridad y en la experiencia profesional, variables que explican las funciones de ingreso individuales (Dirección General para la Cooperación al Desarrollo, 2008).

A partir del trabajo original de Mincer (1974), muchos estudiosos como Card y Krueger (1992), Klenow y Rodríguez-Clare (1997) calcularon una ecuación de salarios en función del número de años de escolaridad y de la experiencia laboral del individuo (ecuación de salarios de Mincer). Las investigaciones de Barro (1991), Mankiw et al. (1992), entre otras, recurrieron a las tasas de escolaridad que dieron como resultado una contribución positiva y significativa del capital humano al crecimiento del producto.

Por su parte, Benhabib y Spiegel (1994) descubrieron una relación no significativa e inclusive negativa para el stock de capital humano (siendo éste la media de años de formación adquirida). Por ende, al estimar el

\footnotetext{
${ }^{1}$ Lo que ocurre cuando en un país el valor de sus importaciones es inferior al de las exportaciones en un determinado periodo. La historia económica del capitalismo de los últimos cinco siglos muestra que la premisa mercantilista se cumple siempre para todos los países, que en un momento histórico dado, han alcanzado el estatuto de economía capitalista desarrollada (Matallana, 2011).
} 
efecto del capital humano sobre el crecimiento del producto, el resultado dependerá de la variable proxy con la cual se esté midiendo el primero. Es necesario resaltar que las variables proxys que se utilizan para medir el capital humano como las mencionadas (tasas de escolaridad o el promedio de ańos de educación adquirida) permiten medir sólo la cantidad más no la calidad de la educación, lo cual podría generar dificultades al momento de realizar comparaciones internacionales entre países (García et al., 2010).

Sería coherente la idea de que la acumulación de capital humano y de formación laboral produzcan incrementos en la productividad en función de la actividad que se realice; sin embargo, el capital humano conseguido por un individuo antes de entrar al mercado laboral no condiciona una adecuada productividad laboral por sí solo, se deben tener en cuenta una serie de condiciones y factores exógenos a la formación adquirida, por ejemplo, la posibilidad de encontrar un trabajo donde pueda desarrollar plenamente sus conocimientos y formación, donde la expectativa respecto a su remuneración sea satisfecha y las motivaciones psicológicas o sociales contribuyan a mejorar su ambiente laboral (Angulo et al., 2012).

Bajo este marco, el presente artículo pretende enriquecer las bases teóricas y empíricas del concepto capital humano, a través de la búsqueda en bibliotecas electrónicas como Dialnet, Jstor, Ebscohost, Redalyc, Scopus, Science Direct, Scielo. Los términos de búsqueda que coadyuvarán a la fundamentación de esta investigación son teoría del capital humano, mercado laboral, oferta laboral, mortalidad y crecimiento económico; cabe señalar que se utiliza como criterio de selección el título del documento y el resumen del mismo. Además, se toman en cuenta investigaciones del Banco Mundial (BM), Banco de la República de Colombia (BRC) y el Departamento Administrativo Nacional de Estadísticas (DANE).

Esta investigación consta de cinco partes, la primera de ellas la constituye este marco introductorio, luego se revisan los distintos fundamentos teóricos del capital humano y la importancia de la variable salud, después se exploran las teorías opuestas al capital humano, posteriormente se presentan algunos hechos estilizados sobre la relación entre el capital humano y la variable salud para varias regiones del mundo y América Latina y, por último, se plantean las conclusiones.

\section{Fundamentos teóricos del capital humano: importancia de la variable salud}

En cuanto a los fundamentos teóricos del capital humano, su contexto histórico y formas de medición, se encuentra que las teorías del capital 
humano argumentan que el conocimiento y la salud determinan el incremento de la productividad individual y el crecimiento económico, es así como Schultz (1960) presentó por primera vez en su Teller Lecture el término capital humano, quien desarrolló su teoría en torno a este concepto a comienzos de los años 60, y luego Becker y Mincer profundizan en ella. No obstante, la hipótesis central es anterior y se encuentra claramente expresada por Adam Smith (1776) en el capítulo diez del libro La riqueza de las naciones, donde escribe que el hombre "que ha sido educado a costa de mucho trabajo y tiempo y debe poder realizar un trabajo que le reembolse el costo de su formación con al menos, los beneficios ordinarios de un capital de igual valor".

Más adelante, en los albores del siglo XX, Irving Fischer (Eicher, 1988) elaboró un concepto de capital que consideraba como tal "todo stock de recursos que permitan originar futuros flujos de ingresos", permitiendo incluir en la inversión tanto la formación de personas como la fabricación de máquinas. Schultz definió que los factores de producción decisivos para el mejoramiento del bienestar de los pobres no son el espacio, la energía y la disponibilidad de tierra cultivable sino el mejoramiento de la calidad de la población, los adelantos en el conocimiento y el mejoramiento de habilidades; a su vez determinó el capital humano como aquel que incluye componentes cualitativos, tales como la habilidad, los conocimientos y atributos similares que afectan la capacidad individual para realizar el trabajo productivo, también planteó que los gastos introducidos para mejorar estas capacidades aumentan el valor de la productividad del trabajo y producirán un rendimiento positivo.

Posteriormente Becker (1964), considerado el sistematizador de los aportes de Schultz, desarrolló formalmente la teoría del capital humano en su libro Human capital. En esencia, su idea básica fue considerar la educación y la formación como inversiones que realizan individuos racionales con el fin de incrementar su eficiencia productiva y sus ingresos. Supuso además que el agente económico (individuo) en el momento que toma la decisión de invertir o no en su educación, arbitra entre los costos de la inversión (por ejemplo, el costo de oportunidad -salario que deja de percibir por estar estudiando-y los costos directos, es decir, los gastos de estudios) y los beneficios que obtendrá en el futuro, si sigue formándose.

El trabajo de Schultz lo continuó Lewis (1980), quien consideró la alimentación y la salud como factores determinantes de la productividad de los trabajadores, por lo cual, las empresas debían velar porque sus trabajadores accedieran a servicios médicos sin contraprestación, tuvieran buenos hábitos alimenticios y, por último, se alojaran en una residencia que les permitiera descansar tranquilamente; estos factores, según Lewis, 
garantizarían el aumento de la productividad. A su vez, la teoría del capital humano expuesta por Mushkin (1962) establece que las personas como agentes productivos mejoran con la inversión en educación y en servicios de salud, y más específicamente, que el incremento de políticas públicas enfocadas en salud aumentan el producto y generan un rendimiento a lo largo de varios ańos; y una medida general de ese rendimiento es el producto del trabajo creado por esta inversión y los ahorros de gastos en salud en el futuro como consecuencia de la reducción de la enfermedad.

De igual forma, la Comisión Económica para América Latina y el Caribe (CEPAL, 1994) consideró que toda sociedad aspira a niveles más elevados de bienestar y de calidad de vida, que entrañen menos muertes infantiles y condiciones propicias para alcanzar vidas más saludables, largas y plenas. A su vez, una fuerza de trabajo sana e instruida puede desplegar plenamente las facultades físicas y mentales que requiere una sociedad para satisfacer sus necesidades.

En la misma línea, Ávila (2009) concluyó que la falta de incentivos para invertir en salud estanca las economías, instalándolas en una trampa de pobreza. Análogamente, Rivera et al. (2008) analizan el papel de la salud como un factor transmisible entre generaciones y sus efectos sobre la acumulación de capital humano y la eficiencia del proceso educativo, apoyando la hipótesis de que el estado de salud individual contribuye a la transmisión intergeneracional de los ingresos, dado que los hijos de padres pobres tienen una mayor tendencia a la enfermedad y acumulan menos capital humano, perpetuando el círculo vicioso de la pobreza.

Por su parte, Grossman (1972) construyó un modelo de capital humano enfocado en el factor salud, en el cual se sustenta que el capital de salud difiere de otras formas de capital; particularmente, sostiene que el stock de conocimiento de una persona afecta su productividad en el mercado así como en el hogar. La productividad de mercado de un individuo estaría representada por su capacidad de producir ganancias en dinero, y por otro lado, su productividad de no mercado estaría reflejada en su capacidad para producir materias primas y productos que aumentan su función de utilidad.

A partir de lo anterior, se establece que la inversión en capital humano, definida por Becker (1964), garantiza aumentos en la productividad de los individuos a partir de aumentos en su stock de conocimientos, mientras que la inversión en capital salud (definida por Grossman) determina la cantidad total de tiempo que puede pasar una persona generando ganancias en dinero y mercancías (aumentando de esta manera su función de utilidad). 
Adicionalmente, Cropper (1977) y Liljas (1998), a partir de las ideas y modelos de Grossman, realizaron modelos de acumulación en capital salud y de demandas de servicios de salud bajo incertidumbre, encontrando otros aspectos importantes como la clasificación de los gastos en servicios médicos con fines preventivos y curativos. A su vez, Rivera y Currais (1999) demuestran que las mejoras en el rubro de salud están relacionadas con un decremento de la tasa de depreciación del capital humano y un incremento de la calidad de la fuerza de trabajo.

En cuanto a la literatura disponible acerca de la relación entre la mortalidad y el capital humano, cabe resaltar el trabajo de Chakraborty y Das (2005) en el cual proponen un mecanismo que une la situación económica y las disparidades en el estado de salud entre los ricos y los pobres. Los autores destacan que la inversión en salud privada mejora la probabilidad de supervivencia hasta el primer periodo de vida y seguidamente, junto con la educación, mejora la productividad laboral de un individuo. Estos autores también resaltan el papel crucial del capital salud en el proceso de desarrollo, ya que a diferencia de otras formas de capital humano, el papel de la salud es único: no sólo genera externalidades positivas, sino que también define el riesgo de mortalidad de los individuos alterando así sus incentivos. Similares conclusiones obtuvieron Beegle (2005) y Sanmartin et al. (2003).

Ahora bien, al distinguir metodologías relacionadas con la medición de impacto económico de la mortalidad, destaca la investigación de Carter et al. (2007) en la que realizan un panel de datos para identificar el impacto económico de las muertes de adultos en edad prematura en Sudáfrica, este estudio permitió advertir que dicho impacto sobre el bienestar económico del hogar es significativamente mayor en aquellas familias que se encuentran por encima de la línea de pobreza, además, que los hogares más pudientes tienden a recuperarse de los impactos económicos con el tiempo, mientras que los menos pudientes no lo hacen. En este sentido, Posada y Rubiano (2007), a partir de revisiones de literatura sobre el tema, concluyeron que los cambios en los retornos al capital humano debidos a choques tecnológicos aumentan la inversión en este factor, mientras que reducen considerablemente las tasas de fertilidad y mortalidad.

Para profundizar en el impacto económico de la mortalidad, Yamauchi et al. (2008) exponen que las muertes de personas trabajadoras de mediana edad generan el aumento del ingreso al mercado de trabajo de adolescentes de mano de obra masculina y femenina, lo que frena la educación de este sector de la población. A su vez, las muertes de personas de mediana edad disminuyen en el futuro la matrícula escolar femenina, lo que su- 
giere que en estos casos las niñas se quedan en casa para cuidar a los enfermos o a la familia en general.

Según esta investigación, en edad adulta el exceso de mortalidad altera la formación de capital humano en la sociedad. Igualmente, Chicoine (2012) examina el efecto que la mortalidad por Síndrome de Inmunodeficiencia Adquirida (Sida) ha causado en los salarios y el empleo en Sudáfrica, dando como resultado que debido a la epidemia, las reducciones en los salarios han sido de 3 a 6\%, y en el empleo de 1,5 a 3,5 puntos porcentuales. Por estas razones concluyeron que la pérdida de ingresos de los hogares en 2007 fue de aproximadamente 4,6 mil millones de dólares, lo que equivale alrededor del 1,7\% del PIB de Sudáfrica en el mismo año.

Cabe resaltar que Barro (1990) elaboró un modelo de crecimiento endógeno y demostró que la inversión en capital humano incluye educación y capacitación laboral; por supuesto, el capital humano y no humano no necesitan ser un sustituto perfecto en la producción. De esta forma, la producción podría mostrar rendimientos a escala en los dos tipos de capital, si se toman en forma conjunta, pero tienen rendimientos decrecientes a escala si se toman de forma separada. Barro (1990) también expone que el producto, aparte del capital físico y del nivel de educación, depende de la cantidad de horas trabajadas y del capital salud de los trabajadores, el cual recoge la productividad de los mismos y la disminución de su ausentismo al lugar de trabajo.

Con respeto al crecimiento económico, Monterubbianesi (2014) describe los principales canales de influencia del estatus de salud sobre el ingreso nacional, a partir de un análisis comparativo y de agrupamiento por tramos sobre la situación entre los diferentes países y regiones. El autor encontró dos canales de influencia: el directo, que se relaciona con salud-productividad y, el indirecto, que se vincula con el hecho de que a mayor salud, menor tasa de depreciación del capital humano y mayor horizonte temporal de vida del individuo, lo que aumentaría la reinversión en capital humano.

Por su parte, Salcedo (2013) realiza un análisis entre la forma neoliberal del capital humano y sus efectos en el derecho a la educación, encontrando que la relación entre economía y educación genera profundos efectos de desigualdad social. Históricamente, la unión entre el capital humano y educación alienta en los habitantes el deseo de aprender. Lo que se muestra como positivo pues contribuye al desarrollo humano; sin embargo, las condiciones socioeconómicas de países neoliberales, que hilvanan su progreso bajo la teoría del capital humano devenido de políticas transnacionales, no están dadas para favorecer el derecho a la educación, pues ésta se ha convertido en una mercancía para la cual todos deben pagar endeudándose. 
Por otro lado, en un estudio empírico realizado para el departamento de Bolívar- Colombia, Pérez y Castillo (2013) demostraron que en los 45 municipios que conforman el departamento, durante el periodo 20022009, las muertes y la oferta laboral inciden en la generación de capital humano cuando esta última es medida con los matriculados en educación básica primaria; es decir, los niños del grupo de entre 5 y 6 años hasta los 11 y 12 años de edad son los más afectados si los padres en edad productiva fallecen o si dejan de integrar la fuerza laboral, debido a que quedarían expuestos a perder el apoyo de la "cabeza del hogar" y, como consecuencia, dejarían de matricularse en la educación básica primaria.

También en Colombia, Castaño et al. (2013) definieron que el nivel de mortalidad es un indicador asociado con la calidad de vida, la seguridad, el nivel de bienestar y el estado de salud de la población. Para estos autores, altas tasas de mortalidad, sobre todo en la población joven, son un buen parámetro para medir la capacidad de relevo que tiene el mercado laboral. De igual manera, más importante aún, es que los altos niveles de mortalidad en la población joven representan a largo plazo una pérdida de capital humano que afecta el consumo y condiciona el crecimiento esperado de la economía (Castaño et al., 2013).

Es importante resaltar que Mincer (1996) planteó que la relación recíproca entre el crecimiento económico y el crecimiento del capital humano es probable que sea un factor importante para el crecimiento económico sostenido, dicho argumento, le sirve de fundamento a Villa (2001) para señalar que dado el nuevo papel de las industrias, basadas en el conocimiento y el énfasis en la innovación, el proceso de trabajo ha tenido un cambio, en donde se ha elevado el valor de las cualidades humanas e intangibles por encima de otros factores productivos que antes eran más importantes.

Asimismo, Carnoy (2006) afirma que los cambios diferenciales en la capacidad de los países para innovar explican en gran medida la brecha de productividad entre las naciones. La educación es importante porque a mayor acumulación de capital humano mayores posibilidades de que el trabajo mejore el proceso de producción y se desarrollen productos nuevos y altamente rentables. El crecimiento lento del conocimiento es una fuerte restricción al progreso, por lo que la mejora en la calidad del factor humano es fundamental para ampliar ideas, objetivos, capacidades y motivaciones favorables al logro económico.

Para cerrar este apartado, es importante hacer un contraste con las teorías contemporáneas del desarrollo de Amartya Sen (2000), quien, siendo uno de los teóricos más influyentes en esta vertiente, en su trabajo Desarrollo y libertad plantea la ampliación de las capacidades y las libertades humanas, y realiza una redefinición del concepto del desarrollo para 
concebirlo como un proceso de expansión de las libertades reales de las que disfrutan los individuos, lo cual contrasta con las visiones más estrictas del desarrollo, como su identificación con el crecimiento del producto nacional bruto, el aumento de las rentas personales, la industrialización, los avances tecnológicos o la modernización social.

Es así que, para Sen, las libertades también dependen de otros determinantes como las instituciones sociales y económicas (por ejemplo, los servicios de educación y de atención médica), así como de los derechos políticos y humanos (entre ellos, la libertad para participar en debates y escrutinios públicos). Es decir, para este autor es importante la educación y la salud como forma de acumulación de capital humano y oportunidades sociales (servicios educativos y sanitarios), pues de esta manera se promueve el desarrollo de las libertades de los individuos.

\section{Teorías opuestas al capital humano}

En este capítulo se destacan las teorías normativas que consideran que la escuela debe servir a los ideales de promover una educación moral dedicada al desarrollo humano y a ideales democráticos, sin referencia a las necesidades del puesto de trabajo. Dewey (1995) es un representante notable de esta concepción considerando que la creación de comunidades sociales especiales en la escuela puede transformar la sociedad adulta sobre similares principios. Este autor rechazó las visiones instrumentales de la escolaridad, según las cuales una actividad educativa específica debía generar un producto educativo específico. Considera que las actividades educativas deben realizarse sólo porque son intrínsecamente valiosas y que la educación progresiva debe centrarse en el niño y no en la preparación para las realidades de la vida adulta. También se opuso al uso de las escuelas con el fin de preparar trabajadores para un orden laboral existente, aunque reconoce que buenas habilidades laborales son un subproducto importante del proceso educativo. En el mismo sentido, Johnes (1997) señala que la educación existe porque aporta una utilidad.

Por su parte, De la Fuente (2002) destaca que la evidencia empírica no siempre ha sido consistente. Las variables educativas son con frecuencia no significativas o incluso entran con el signo equivocado en regresiones de crecimiento, especialmente cuando éstas se estiman utilizando especificaciones en diferencias o con técnicas de panel. Conclusiones similares obtuvieron Mulligan y Sala-I-Martin (2000) al construir un panel de datos de los índices de capital humano de los estados de Estados Unidos para el periodo 1940-1990, encontrando que el stock de capital humano creció dos veces más rápido que el promedio de años de escolaridad, y que la desigualdad de capital humano entre los estados de ese 
país, aumentó durante la década de 1980, mientras que la desigualdad de la educación disminuyó durante el mismo periodo.

Igualmente, al examinar la relación entre el grado de escolarización y el crecimiento, los resultados no han sido siempre consistentes ni los esperados en relación con los planteamientos teóricos, por lo que se ha fomentado un creciente escepticismo sobre el papel de la escolarización en el proceso de crecimiento, e incluso ha llevado a algunos autores como Pritchett (1999) a considerar seriamente las posibles razones por las que la inversión educativa podría no contribuir al crecimiento de la productividad.

El punto de partida de los estudios resumidos anteriormente fue la sospecha de que la deficiente calidad de los datos es una de las principales razones por las que diversos estudios recientes no han encontrado una relación significativa entre capital humano y crecimiento económico. Autores como Krueger y Lindhal (2001), Kumar (2006), así como Cohen y Soto (2007) apoyan esta hipótesis.

Consecuente con lo anterior, al momento de comprender las nociones y tratar de entender los fundamentos del capital humano, es importante analizar la interacción existente entre la distribución del capital humano, el progreso tecnológico y el crecimiento económico; variables que se consideran en la investigación de Galor y Tsiddon (1997), en la cual proponen que la composición del capital humano es un factor importante en la determinación del modelo de desarrollo económico. El estudio demuestra que el patrón evolutivo de la distribución del capital humano, la distribución del ingreso y el crecimiento económico se determinan simultáneamente por la interacción entre las externalidades.

De este modo, la economía puede resultar beneficiada si subvenciona la educación de un grupo selecto de individuos que a la larga generan externalidades suficientes para sacar a la sociedad en su conjunto a un estado de igualdad y prosperidad. Por otra parte, una economía que prematuramente implementa una política destinada a mejorar la igualdad en la distribución educativa puede ser atrapada innecesariamente en un equilibrio de bajo gasto sin llegar nunca a la prosperidad.

Asimismo, en otra referencia para España, Sáez (2002) pasa revista a determinados fenómenos relacionados con la adecuación entre capital humano y los requerimientos de empleo del sistema productivo, en una etapa del crecimiento económico español donde la innovación es el eje que determina las posibilidades competitivas del sistema productivo. Así, el autor encuentra que el potencial de recursos humanos en Espańa, de cara a las tendencias de creciente tecnificación en los procesos de producción que afectan a la economía internacional, presenta notables diferencias respecto a los países de la Unión Europea, centradas básicamente en una distribución desequilibrada entre niveles de cualificación inicial, en claro 
detrimento del grupo de personas de preparación técnico-profesional intermedio; grupo que proporciona habitualmente la base de calidad y productividad de todo sistema productivo moderno.

Los requerimientos del sistema productivo condicionan el ritmo de absorción de los recursos humanos disponibles en el mercado de trabajo, lo que, en el caso de quienes disponen de una titulación universitaria, parece claro. Se genera así una diferencia importante entre las tasas de empleo efectivo de las distintas carreras, que se va atenuando a medida que pasa el tiempo, debido en buena medida a la versatilidad de las personas para desempeñar determinados puestos de trabajo de naturaleza diversa dentro del sistema productivo.

$\mathrm{Al}$ margen de ello, aquellas personas que responden mejor a las necesidades de las empresas tienen, lógicamente, una tasa de incorporación más alta; factores tales como la capacidad para utilizar técnicas relacionadas con las tecnologías de la información y las comunicaciones (TICs) resultan claramente favorables, comprobándose paralelamente que el acceso a puestos cualificados se ve también influido por la carrera cursada, el manejo apropiado de idiomas y el conocimiento de técnicas de computación y otras relacionadas con la informática.

\section{Capital humano y variable salud: hechos estilizados para varias regiones del mundo y América Latina}

A continuación se realiza un análisis comparativo mostrando algunos hechos estilizados de la relación entre capital humano y salud en varias regiones del mundo y en los países de América Latina, los cuales se convierten en evidencia empírica que demuestra la validez de los postulados teóricos acerca de la relación entre estas dos variables.

Como se observa en el cuadro 1, existen grandes diferencias en cuanto al indicador de capital humano a nivel regional, dado que mientras territorios como África subsahariana y sudeste de Asia presentan niveles significativamente bajos en el indicador de capital humano, otras regiones como Europa occidental y América del Norte exhiben niveles significativamente mayores, lo que implica que se está lejos de la convergencia regional en términos de capital humano. Por su parte, el mínimo desempeño mostrado por las regiones que presentan las menores cifras en el indicador del capital humano, es consecuente con que poseen los registros más bajos en indicadores como salud y experiencia comparándolos con las demás regiones del mundo. 


\section{Cuadro 1 \\ Indicadores de salud, experiencia y capital humano en varias regiones del mundo (2000)}

\begin{tabular}{lccccc}
\hline & Salud & $\begin{array}{c}\text { Salud/ } \\
\text { promedio } \\
\text { mundial }\end{array}$ & Experiencia & $\begin{array}{c}\text { Experiencial } \\
\text { promedio } \\
\text { mundial }\end{array}$ & $\begin{array}{c}\text { Indicador } \\
\text { de capital } \\
\text { humano }\end{array}$ \\
\hline África subsahariana & 47,91 & 0,72 & 10,97 & 0,71 & $-4,57$ \\
Sudeste de Asia & 61,21 & 0,92 & 12,99 & 0,85 & $-2,65$ \\
Oriente medio y norte de & 72,21 & 1,08 & 13,42 & 0,87 & $-0,04$ \\
África & & & & & \\
América Latina y el Caribe & 70,81 & 1,06 & 14,52 & 0,94 & 0,19 \\
Este de Asia y el Pacífico & 71,59 & 1,07 & 16,43 & 1,07 & 1,89 \\
Europa del este y Asia central & 71,09 & 1,06 & 19,61 & 1,28 & 2,74 \\
Europa occidental & 77,74 & 1,16 & 21,95 & 1,43 & 5,33 \\
América del Norte & 77,97 & 1,17 & 18,57 & 1,21 & 6,3 \\
Promedio de los países & 66,67 & 1 & 15,37 & 1 & 0,33 \\
\hline
\end{tabular}

Fuente: elaboración propia con base en Giménez (2005).

Seguidamente, en el tercer lugar se encuentra el Oriente Medio y norte de África cuyas características se asemejan a América Latina y el Caribe; no obstante, esta última presenta una cantidad superior en cuanto a experiencia, hecho que le otorga un índice de capital humano más elevado. Además, para las regiones como el este de Asia, el Pacífico, Europa del este y Asia central, el índice de capital humano comienza a mostrar valores significativamente positivos.

Cerrando el análisis de los indicadores de capital humano y de salud en términos regionales, se encuentra que Europa occidental presenta el valor más alto en cuanto a experiencia, y América del Norte posee el primer lugar en el indicador de capital humano dado que cuenta con los datos más altos en salud.

En términos de la relación entre la salud y el índice de capital humano a nivel regional, cabe destacar el aporte positivo que hace este rubro al índice, dado que las regiones que presentan mayores niveles en términos de salud, también son las que obtuvieron los índices de capital humano más altos, lo que sugiere la gran importancia de la salud. No obstante, nuevamente se evidencia la divergencia en términos regionales por cuenta de la salud, dado que mientras regiones como África subsahariana, sudeste de Asia, Oriente Medio y norte de África alcanzaron 47,91; 61,21 y 72,21, respectivamente, regiones más avanzadas como Europa occidental y América del Norte obtuvieron 77,74 y 77,97, respectivamente.

Por su parte, al analizar más específicamente el indicador de capital humano en los países que conforman la región de América Latina y el 
Caribe, como se aprecia en el cuadro 2, es posible observar grandes brechas al interior de la región dado que existen países como Barbados, Uruguay, Chile y Trinidad y Tobago con indicadores de capital humano de 3,61; 2,$75 ; 2,72$ y 2,28, respectivamente, que contrastan con países como Guatemala, Honduras, Nicaragua y Paraguay que presentan -3,34; -2,16; $-1,93$ y $-1,33$, respectivamente.

\section{Cuadro 2}

\section{Indicadores de salud, experiencia y capital humano en América Latina y El Caribe (2000)}

\begin{tabular}{lrrrcr}
\hline & Salud & $\begin{array}{c}\text { Salud/ } \\
\text { promedio } \\
\text { mundial }\end{array}$ & Experiencia & $\begin{array}{c}\text { Experiencial } \\
\text { promedio } \\
\text { mundial }\end{array}$ & $\begin{array}{c}\text { Indicador } \\
\text { de capital } \\
\text { bumano }\end{array}$ \\
\hline Argentina & 73,57 & 1,04 & 17,31 & 1,19 & 2,07 \\
Barbados & 75,66 & 1,07 & 20,72 & 1,43 & 3,61 \\
Bolivia & 62,06 & 0,88 & 11,79 & 0,81 & $-2,08$ \\
Brasil & 67,15 & 0,95 & 15,62 & 1,08 & 0,12 \\
Colombia & 70,35 & 0,99 & 14,07 & 0,97 & $-0,53$ \\
Costa Rica & 76,84 & 1,09 & 14,59 & 1,01 & 0,81 \\
Chile & 75,51 & 1,07 & 16,35 & 1,13 & 2,72 \\
Ecuador & 69,23 & 0,98 & 13,6 & 0,94 & $-0,38$ \\
El Salvador & 69,53 & 0,98 & 13,66 & 0,94 & $-0,84$ \\
Guatemala & 64,89 & 0,92 & 10,72 & 0,74 & $-3,34$ \\
Guyana & 63,7 & 0,9 & 14,95 & 1,03 & $-0,14$ \\
Honduras & 69,82 & 0,99 & 10,88 & 0,75 & $-2,16$ \\
Jamaica & 75,16 & 1,06 & 15,29 & 1,05 & 0,54 \\
México & 72,14 & 1,02 & 14,23 & 0,98 & 0,58 \\
Nicaragua & 68,63 & 0,97 & 10,94 & 0,75 & $-1,93$ \\
Panamá & 73,88 & 1,04 & 14,77 & 1,02 & 1,23 \\
Paraguay & 69,95 & 0,99 & 11,5 & 0,79 & $-1,33$ \\
Perú & 68,74 & 0,97 & 13,71 & 0,94 & 0,08 \\
Trinidad y Tobago & 72,63 & 1,03 & 17,48 & 1,2 & 2,28 \\
Uruguay & 74,3 & 1,05 & 19,16 & 1,32 & 2,75 \\
Venezuela & 73,16 & 1,03 & 13,53 & 0,93 & $-0,06$ \\
Promedio de los países & 70,81 & 1 & 14,52 & 1 & 0,19 \\
\hline & & & & &
\end{tabular}

Fuente: elaboración propia con base en Giménez (2005).

Con el fin de analizar el comportamiento del indicador de capital humano de estos países y su relación con la variable salud, cabe destacar que precisamente los países que poseen los índices de capital humano más elevados son los que mayores valores en términos de salud presentan. En la figura 1 se aprecia la relación entre la salud y el indicador de capital humano para los cuatro países que ocupan los primeros lugares en el 
índice mencionado y los cuatro de peor desempeño del mismo. Se evidencia no sólo la relación positiva existente entre ambas variables dada la línea de tendencia, sino también la alta correlación que éstas presentan.

\section{Figura 1}

Dispersión del indicador de capital humano y salud para los cuatro países más altos y los cuatro países más bajos en el indicador de capital humano

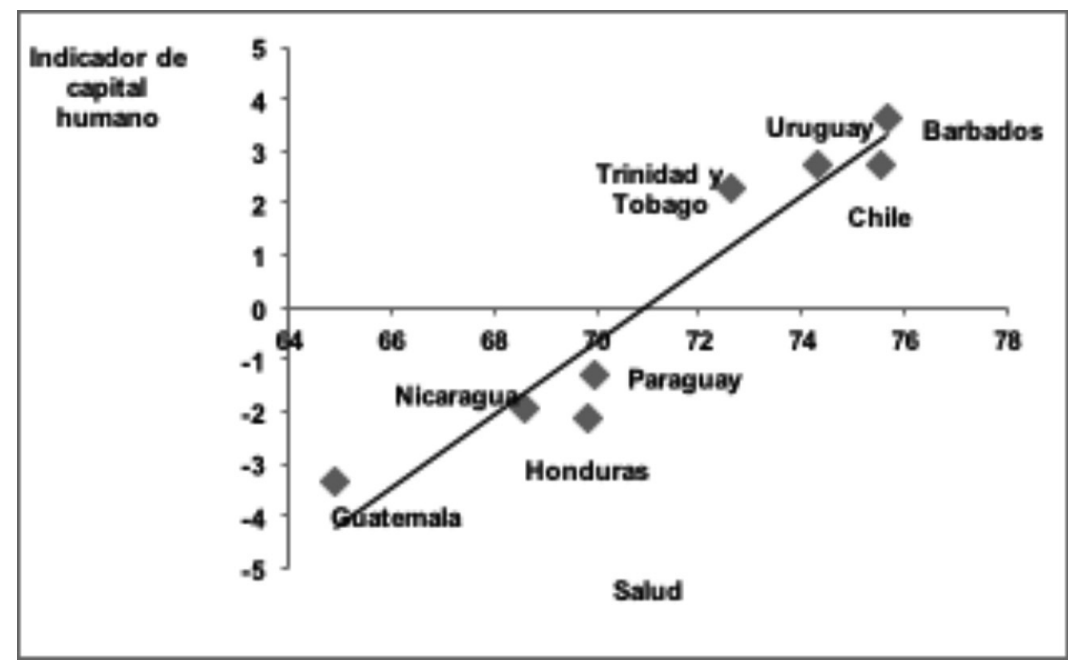

Fuente: elaboración de autores con base en Giménez (2005).

Por último, muestra la evolución del indicador de capital humano en el periodo comprendido entre 1960 y 2000 con una periodicidad quinquenal. En éste se revela que países como Brasil, Chile, Colombia, Costa Rica, Ecuador, El Salvador, Honduras, México, Nicaragua, Panamá, Perú y Venezuela han tenido mejoras notables a lo largo de los 40 años, dado que el crecimiento en términos de capital humano se ha incrementado paulatinamente; en contraste, países como Argentina, Paraguay y Uruguay han tenido retrocesos en esta variable. Finalmente, Bolivia y Guatemala mantienen comportamientos constantes en sus indicadores de capital humano. 


\section{Cuadro 3}

\section{Evolución del indicador de capital humano en América Latina y El Caribe (1960-2000)}

\begin{tabular}{lrrrrrrrrr}
\hline & 1960 & 1965 & 1970 & 1975 & 1980 & 1985 & 1990 & 1995 & 2000 \\
\hline Argentina & 3,77 & 3,92 & 4,07 & 3,16 & 2,78 & 2,71 & 2,42 & 2,29 & 2,07 \\
Bolivia & $-2,02$ & $-2,25$ & $-2,64$ & $-2,61$ & $-2,49$ & $-2,31$ & $-2,02$ & $-2,07$ & $-2,08$ \\
Brasil & $-1,31$ & $-1,17$ & $-0,84$ & $-0,7$ & $-0,61$ & $-0,28$ & $-0,18$ & $-0,05$ & 0,12 \\
Colombia & $-1,71$ & $-1,68$ & $-1,27$ & $-0,46$ & $-0,33$ & 0,17 & $-0,3$ & $-0,38$ & $-0,53$ \\
Costa Rica & $-1,35$ & $-0,95$ & $-0,09$ & 0,77 & 0,83 & 1,16 & 0,8 & 0,91 & 0,81 \\
Chile & 0,67 & 0,79 & 1,4 & 1,69 & 2,15 & 2,33 & 2,24 & 2,32 & 2,72 \\
Ecuador & $-1,73$ & $-1,77$ & $-1,74$ & $-1,36$ & $-0,79$ & $-0,36$ & $-0,26$ & $-0,3$ & $-0,38$ \\
El Salvador & $-2,78$ & $-2,7$ & $-2,3$ & $-2,33$ & $-2,1$ & $-1,64$ & $-1,32$ & $-1,04$ & $-0,84$ \\
Guatemala & $-3,35$ & $-3,26$ & $-3,18$ & $-3,27$ & $-3,34$ & $-3,6$ & $-3,45$ & $-3,46$ & $-3,34$ \\
Honduras & $-3,56$ & $-3,6$ & $-3,58$ & $-3,31$ & $-3,25$ & $-2,34$ & $-2,26$ & $-2,24$ & $-2,16$ \\
México & $-1,76$ & $-1,65$ & $-1,34$ & $-1,06$ & $-0,66$ & $-0,31$ & 0,37 & 0,55 & 0,58 \\
Nicaragua & $-3,38$ & $-3,27$ & $-2,97$ & $-2,94$ & $-3,12$ & $-2,99$ & $-2,52$ & $-2,05$ & $-1,93$ \\
Panamá & 0,06 & 0,35 & 0,28 & 0,55 & 0,97 & 1,35 & 1,47 & 1,31 & 1,23 \\
Paraguay & $-0,47$ & $-0,77$ & $-0,59$ & $-0,6$ & $-0,79$ & $-1,1$ & $-1,02$ & $-1,28$ & $-1,33$ \\
Perú & $-2,34$ & $-2,18$ & $-1,71$ & $-1,39$ & $-0,8$ & $-0,53$ & $-0,47$ & $-0,18$ & 0,08 \\
Uruguay & 4,79 & 4,73 & 4,69 & 3,86 & 3,46 & 3,54 & 3,25 & 2,77 & 2,75 \\
Venezuela & $-1,4$ & $-1,13$ & $-0,73$ & $-0,22$ & 0,6 & 0,7 & 0,11 & 0,27 & $-0,06$ \\
Promedio & $-1,05$ & -0.98 & -0.74 & -0.60 & -0.44 & $-0,21$ & $-0,18$ & $-0,15$ & $-0,13$ \\
de los países & & & & & & & & & \\
\hline
\end{tabular}

Fuente: elaboración propia con base en Giménez (2005).

\section{Conclusión}

De acuerdo con la literatura existente, las primeras aproximaciones al término capital humano se dan en el libro La riqueza de las naciones de Adam Smith, quien propone que el hombre que ha sido educado a costa de mucho trabajo y tiempo, debe poder ocuparse en una actividad que le reembolse el costo de su formación con, al menos, los beneficios ordinarios de un capital de igual valor.

Más adelante Schultz (1960) plantea que los factores de producción decisivos para el mejoramiento del bienestar son el incremento de la calidad de la población, los adelantos en el conocimiento y el perfeccionamiento de habilidades. Luego, Becker (1964) desarrolla formalmente la teoría del capital humano argumentando que la educación y la formación son inversiones que realizan los individuos racionales con el fin de incrementar su eficiencia productiva y sus ingresos.

Posteriormente, Lewis (1980) aporta los componentes alimentación y salud como factores determinantes de la productividad de los trabaja- 
dores, siendo coherente con Mushkin (1962), quien sostiene que las mejoras en salud aumentan el producto y continúan generando un rendimiento a lo largo de varios años. Por su parte, Grossman (1972) desarrolla un modelo de capital salud en el que determina la cantidad total de tiempo que puede pasar una persona generando ganancias en dinero y mercancías (lo que aumenta su función de utilidad). De igual manera, Cropper (1977) y Liljas (1998), a partir de ideas de Grossman (1972), realizan modelos de acumulación en capital salud, y posteriormente Barro (1990) elabora un modelo de crecimiento económico endógeno demostrando que la inversión en capital humano incluye educación y capacitación laboral; luego Mincer (1958) y Johnes (1997) plantean la relación recíproca entre el crecimiento económico y el crecimiento del capital humano, lo que se convierte en un factor importante para el crecimiento económico sostenido.

Por otro lado, en cuanto a las metodologías que se trabajan en mediciones de capital humano, diversos autores encuentran que la evidencia empírica no siempre ha sido consistente. Las variables educativas son con frecuencia no significativas o incluso entran con el signo equivocado en regresiones de crecimiento, especialmente cuando éstas se estiman utilizando especificaciones en diferencias o con técnicas de panel.

La literatura disponible sobre la relación entre la mortalidad y el capital humano propone mecanismos que unen la situación económica y las disparidades en el estado de salud entre los ricos y los pobres; demuestra que la desigualdad inicial de la salud puede ser un factor clave para explicar la persistencia observada en la riqueza y la desigualdad de ingresos entre los hogares; es decir, se resalta el papel crucial del capital salud en el proceso de desarrollo.

El papel de la salud es único debido a que no sólo genera externalidades positivas, sino también define el riesgo de mortalidad de los individuos, alterando así sus incentivos. Además, uno de los impactos más representativos se da por el lado del capital humano y el mercado laboral, ya que el capital humano perdido con la muerte prematura de un adulto es irrecuperable y tiene un efecto negativo sobre el mercado laboral, encontrándose que las horas de trabajo que deja de realizar un adulto fallecido, en algunos casos las substituyen los nińos del hogar y en otros casos el hogar se vuelve más propenso a recibir un co-residente que asume esas horas.

Las muertes de trabajadores de mediana edad aumentan la oferta laboral de adolescentes. A su vez, en el futuro disminuyen la matrícula escolar femenina, lo que sugiere que las nińas se quedan en casa para cuidar a los enfermos o a la familia en general, y que las mujeres adultas tienden a convertirse en una parte de la fuerza laboral posterior a la muerte de los hombres adultos en edad productiva. 
Finalmente, los hechos estilizados de la relación entre capital humano y salud en varias regiones del mundo y en los países de América Latina demuestran la validez de los postulados teóricos acerca de la relación entre estas dos variables. Pues existen grandes diferencias en cuanto al indicador de capital humano a nivel regional, dado que mientras territorios como África subsahariana y sudeste de Asia presentan niveles significativamente bajos en el indicador, otras regiones como Europa occidental y América del Norte exhiben niveles significativamente mayores, lo que implica que se está lejos de la convergencia regional en términos de capital humano. Además, la evidencia no sólo muestra la relación positiva existente entre ambas variables sino también la alta correlación que éstas presentan.

\section{Fuentes consultadas}

Angulo, Grace, Raúl Quejada y Martha Yánez (2012), “Educación, mercado de trabajo y satisfacción laboral: el problema de las teorías del capital humano y señalización de mercado", Revista de la Educación Superior, XLI (3), Asociación Nacional de Universidades e Instituciones de Educación Superior, México, pp. 51-66.

Ávila, Óscar (2009), Salud y crecimiento económico: un modelo de generaciones traslapadas, expectativa de vida endógena y capital humano, Documento de Trabajo núm. 60, Universidad del Rosario, Bogotá, pp. 1-36.

Barro, Roberto (1991), "Economic growth in a cross section of countries", Quarterly Journal of Economics, 106 (2), Oxford University Press, Oxford, pp. 407-443.

Barro, Roberto (1990), "Government spending in a simple model of endogeneous growth", Journal of Political Economy, 98 (5), The University of Chicago Press, Chicago, pp. 103-125.

Becker, Gary (1964), Human capital: a theoretical and empirical analysis, with special reference to education, National Bureau of Economic Research, Londres.

Beegle, Kathleen (2005), "Labor effects of adult mortality in Tanzanian households", Economic Development and Cultural Change, 53 (3), The University of Chicago Press, Chicago, pp. 655-683. 
Benhabib, J. y Spiegel, M. (1994), "The role of human capital in economic development: evidence from aggregate cross-country data", Journal of Monetary Economics, 34 (2), New York University, Nueva York, pp. 143-147.

Card, David, y Alan Krueger (1992), "Does school quality matter? returns to education and the characteristics of public schools in the United States", Journal of Political Economy, 100 (1), The University of Chicago Press, Chicago, pp. 1-40.

Carnoy, Martin (2006), Economía de la educación, Universitat Oberta de Catalunya, Barcelona.

Carter, Michael, Julian May, Jorge Agüero y Sonia Ravindranath (2007), "The economic impact of premature adult mortality: panel data evidence from KwaZulu-natal, South Africa", Official Journal of the international AIDS Society, 21 (7), International AIDS Society, Ginebra, pp. 67-73.

Castaño, Andrés, Juan, Correa, Luis Alvis y Nelson Alvis, (2013), "Valoración económica de la mortalidad en la región Caribe de Colombia, 2004-2008”, Semestre Económico, 16 (33), Universidad de Medellín, Medellín.

Chakraborty, Shankha y Mausumi Das (2005), "Mortality, human capital and persistent inequality", Journal of Economic Growth, 10 (2), Springer International Publishing, Nueva York, pp. 159-192.

Chicoine, Luke (2012), "AIDS mortality and its effect on the labor market: evidence from South Africa", Journal of Development Economics, 98 (2), Elsevier, Amsterdam, pp. 256-269.

Cohen, Daniel y Marcelo Soto (2007), "Growth and human capital: good data, good results", Journal of Economic Policy Research, 12 (1), Springer International Publishing, Nueva York, pp. 51-76.

CEPAL (Comisión Económica para América Latina y el Caribe) (1994), Salud, equidad y capital humano en el Itsmo Centroamericano, CEPAL, Santiago de Chile. 
Cropper, Maureen (1977), Health, investment in health, and occupational choice, Journal of Political Economy, 85 (6), Univerity of Chicago Press, Chicago, pp. 1273-1294.

De la Fuente, Ángel (2002), "Capital humano y crecimiento: nuevas series de escolarización y algunos resultados para la OCDE" Economía industrial, 6 (348), Ministerio de Industria, Turismo y Comercio, Madrid, pp. 41-42.

Dewey, John (1995), Democracia y educación, Madrid, Editorial Morata.

Dirección General para la Cooperación al Desarrollo (2008), Capital bumano, recurso para el desarrollo, Ministerio de Asuntos Exteriores italiano, Milán.

Domínguez, Marcos (2004), "El papel de la fisiocracia en nuestros días: una reflexión sobre el análisis económico de los recursos naturales y el medio ambiente", Revista Galega de Economía, 13 (1-2), Universidad de Santiago de Compostela, Santiago de Compostela, pp. 1-12.

Eicher, Jean Claude (1988), “Treinta años de economía de la educación”, Ekonomiaz: Revista Vasca de Economía, núm. 12, Eusko Jaurlaritza Gobierno Vasco, Vitoria Gasteiz, pp. 11-38.

Galor, Oded y Daniel Tsiddon (1997), "The distribution of human capital and economic growth", Journal of Economic Growth, 2 (1), Kuwer Academic Publisher, Dordrecht, pp. 93-124.

García, Josefina, Juan Gómez y José Solana (2010), “La medición del capital humano", Anales de Economía Aplicada, Asociación Española de Economía Aplicada, Universidad de La Rioja, Logroño, pp. 2-5.

Giménez, Gregorio (2005), "La dotación de capital humano de América Latina y el Caribe", Revista de la CEPAL, núm. 86, CEPAL, Santiago de Chile, pp. 103-122.

Grossman, Michael (1972), "On the concept of health capital and the demand for health, Journal of Political Economy, 80 (2), The University Chicago Press, Chicago, pp. 223-255. 
Johnes, Geraint (1997), "Economía de la educación: capital humano, rendimiento educativo y mercado de trabajo", Revista Española de Investigaciones Sociológicas (77), Centro de Investigaciones Sociológicas, Madrid, pp. 370-377.

Klenow, Petery Rodríguez-Clare, Andrés (1997), “The neoclassical revival in growth economics: has it gone too far?", National Bureau of Economic Research Macroeconomics Annual, vol. 12, MIT Press, pp. 73-114.

Krueger, Alan y Mikael Lindhal (2001), "Education for growth: why and for whom?", Journal of Economic Literature, 39 (4), American Economic Association, Nashville, pp. 1101-1136.

Kumar, Chandra S. (2006), "Human capital and growth empirics", Journal of Developing Areas, 40 (1), College of Business, Tennessee State University, Tennessee, pp. 153-179.

Lewis, Arthur (1980), "The slowing down of the engine of growth", The American Economic Review, 70 (4), American Economic Association, Nashville, pp. 555-564.

Liljas, Bengt (1998), The demand for health with uncertainty and insurance, Journal of Health Economics, 17 (2), Cornell University, Ithaca, pp. 153-170.

Mankiw, Gregory, David Romer y David Weil, (1992), “A contribution to the empirics of economic growth", The Quarterly Journal of Economics, 107 (2), Oxford University Press, Oxford, pp. 407-437.

Matallana, Hernando (2011), "Mercantilismo, acumulación de capital y desarrollo económico en la economía monetaria de producción (Nacional)", Cuadernos de Economía, XXX (55), Universidad Nacional de Colombia, Bogotá, pp. 1-29.

Mincer, Jacob (1996), "Economic development, growth of human capital, and the dynamics of the wage structure", Journal of Economic Growth, 1 (1), Kuwer Academic Publisher, Boston, pp. 29-48.

Mincer, Jacob (1974), Schooling, experience and earnings, National Bureau of Economic Research, Cambridge. 
Mincer, Jacob (1958), "Investment in human capital and personal income distribution", Journal of Political Economy, 66 (4), University Chicago Press, Chicago, pp. 281-302.

Monterubbianesi, Pablo Daniel (2014), "Salud y crecimiento económico: influencias teóricas y vinculaciones empíricas", Revista de Ciencias Sociales (Cr), I (143), Universidad de Costa Rica, San José Costa Rica, pp. 131-140.

Mulligan, Casey y Xavier Sala-I-Martin (2000), “Measuring aggregate human capital", Journal of Economic Growth, 5 (3), Springer International Publishing, pp. 215-252.

Mushkin, Selma (1962), "Investment in human beings", Journal of Political Economy, 70 (5), The University Chicago Press, Chicago, pp. 129-157.

Pérez, Dewin y Jorge Castillo (2013), "Incidencias de las muertes y la oferta laboral en la generación de capital humano en el departamento de Bolívar", Revista de Economía del Caribe, núm. 12, Universidad del Norte, Barranquilla, pp. 74-105.

Posada, Carlos y Eliana Rubiano (2007), El crecimiento económico internacional en la segunda mitad del siglo XX: ¿qué factores lo determinaron?, Banco de la República, Bogotá.

Pritchett, Lant (1999), "Where has all the education gone?", World Bank Economic Review, 15 (3), Oxford University Press, Oxford, pp. 367-391.

Quezada, Alberto (2011), "Una visión crítica del modelo económico dominante desde la perspectiva de los sitemas complejos", Revista Análisis Económico, XXVI (63), Universidad Autónoma Metropolitana unidad Azcapotzalco, México, pp. 37-49.

Rivera, Berta, Luis Currais y Paolo Rungo (2008), "La transmisión de las desigualdades en el estado de salud: efectos sobre la equidad intergeneracional a partir de la acumulación de capital humano", Cuadernos Económicos de ICE, núm. 75, Universidad de la Rioja, Logroño, pp. 99-114. 
Rivera, Berta y Luis Currais (1999), "Convergencia y capital humano: una aproximación empírica", Cuadernos de Estudios Empresariales, núm. 9, Universidad Complutense de Madrid, Madrid, pp. 249-260.

Sáez, Felipe (2002), "Capital humano, innovación y empleo. Su adecuación en el caso Español", Economía industrial, 6 (348), Ministerio de Ciencia y Tecnología, Madrid, pp. 67-76.

Salcedo, Javier (2013), "La forma neoliberal del capital humano y sus efectos en el derecho a la educación", Revista Actualidades Pedagógicas, núm. 61, Universidad de La Salle, Bogotá, pp. 113-138.

Sanmartin, Claudia, Nancy Annette Ross, S. Tremblay, M. Wolfson, James R. Dunn y John Lynch (2003), "Labour market income inequality and mortality in North American metropolitan areas", Journal of Epidemiology and Community Health, 57 (10), National Library of Medicine, Bathesda, pp. 792-797.

Schultz, Theodore W. (1960), "Capital formation by Education”, Journal of Political Economy, 68 (6), The University Chicago Press, Chicago, pp. 571-583.

Sen, Amartya (2000), Desarrollo y libertad, Editorial Planeta, Buenos Aires.

Smith, Adam (1776), Investigación de la naturaleza y causas de la riqueza de las naciones, Alianza, Madrid.

Villa Arcila, Carlos Leonardo (2001), Economía de la educación con énfasis en la educación superior, Universidad de los Andes, Bogotá.

Yamauchi, Futoshi, Thabani Buthelezi y Myriam Velia (2008), "Impact of prime-age adult mortality on labor supply evidence from adolescents and women in South Africa", HIV, Livelihoods, Food and Nutrition on Security: Findings from renewal research (20072008), núm. 15, International Food Policy Research Institut, Washington, pp. 15-16. 
Recibido: 29 de junio de 2015. Corregido: 18 de diciembre de 2015. Aceptado: 2 de marzo de 2016.

Dewin Iván Pérez-Fuentes. Colombiano. Magíster en ciencias económicas por la Universidad Nacional de Colombia, estudiante de doctorado en ciencias económicas de la Universidad del Zulia, Venezuela y economista de la Universidad de Cartagena, Colombia. Actualmente es profesor-investigador y director del Programa de Economía de la Universidad de Cartagena, Colombia. Es miembro director-investigador del Observatorio del Mercado Laboral de Cartagena y Bolívar, Colombia. Su línea de investigación actual es mercado laboral y desarrollo industrial. Entre sus últimas publicaciones destacan: "Participación femenina en el mercado laboral de Cartagena, 2008-2013”, Economía \& Región, 7 (2), Universidad Tecnológica de Bolívar, Cartagena, pp. 5-29 (2014); en coautoría: "Incidencias de las muertes y la oferta laboral en la generación de capital humano en el departamento de Bolívar", Revista de Economía del Caribe, núm. 12, Universidad del Norte, Barranquilla, pp. 74-105 (2013); "Liberalización, reformas y empleo en la industria manufacturera del Caribe Colombiano 1974-2004”, Investigación \& Desarrollo, 18 (1), Universidad del Norte, pp. 142-161 (2010).

Jorge Leonardo Castillo-Loaiza. Colombiano. Economista de la Universidad de Cartagena y candidato a magíster en economía aplicada de la Universidad EAFIT, Medellín, Colombia. Actualmente es líder del Sistema de Investigación, Desarrollo Tecnológico e Innovación (SENNOVA), Colombia. Exbecario del Programa Jóvenes-Investigadores Innovadores "Virginia- Gutiérrez de Pineda" (Colciencias), Colombia. Su línea de investigación actual es capital humano, innovación y mercado laboral. Entre sus últimas publicaciones destacan; en coautoría: "Incidencias de las muertes y la oferta laboral en la generación de capital humano en el departamento de Bolívar", Revista de Economía del Caribe, núm. 12, Universidad del Norte, Barranquilla, pp. 74-105 (2013). 